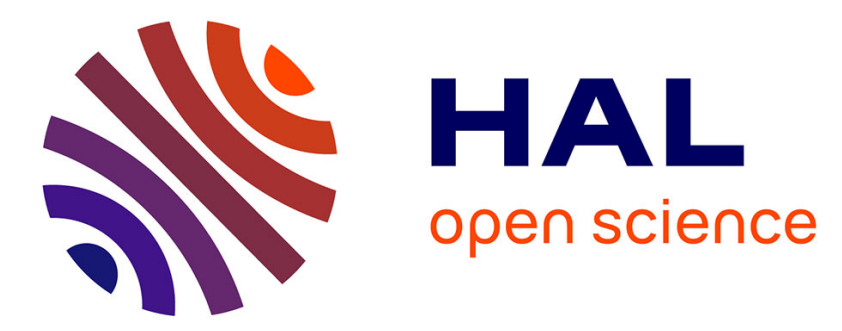

\title{
Un casque antique en bronze au Musée de Chalon-sur-Saône
}

Louis Armand-Calliat

\section{To cite this version:}

Louis Armand-Calliat. Un casque antique en bronze au Musée de Chalon-sur-Saône. Gallia - Fouilles et monuments archéologiques en France métropolitaine, 1965, 23 (2), pp.261-266. 10.3406/galia.1965.2418. hal-01933691

\section{HAL Id: hal-01933691 \\ https://hal.science/hal-01933691}

Submitted on 26 Feb 2020

HAL is a multi-disciplinary open access archive for the deposit and dissemination of scientific research documents, whether they are published or not. The documents may come from teaching and research institutions in France or abroad, or from public or private research centers.
L'archive ouverte pluridisciplinaire HAL, est destinée au dépôt et à la diffusion de documents scientifiques de niveau recherche, publiés ou non, émanant des établissements d'enseignement et de recherche français ou étrangers, des laboratoires publics ou privés.

\section{(ㅇ)(1) $\$$}

Distributed under a Creative Commons Attribution - NonCommercial - NoDerivatives| 4.0 


\section{Un casque antique en bronze au Musée de Chalon-sur-Saône}

Durant l'été 1963, le Musée de Chalon a pu faire l'acquisition d'un casque de bronze qui était entre les mains d'un dragueur'. Cet ouvrier l'avait retiré, peu auparavant, des sables du Doubs, dans l'arrondissement de Chalon, sur la commune de Ciel, à la hauteur de l'écart du Chapot. Il s'agit, croyons-nous, d'une pièce assez peu commune pour mériter une mention particulière.

Sa forme générale est des plus simples (fig. 1). Ce n'est qu'une calotte métallique faite d'une seule plaque martelée, dépourvue de tout ornement, longue de $0,240 \mathrm{~m}$, large de $0,173 \mathrm{~m}$, haute de $0,135 \mathrm{~m}$; elle épouse très exactement les contours du crâne et comporte seulement un couvre-nuque compris dans ces dimensions dont la largeur est de 0,033, au maximum.

Ce qui frappe tout d'abord, quand on examine cette arme défensive, c'est la minceur, en certains points, de la paroi métallique. Sans doute, vers le front et la nuque, elle atteint bien de 3 à 4 mm environ, mais sur le sommet, elle se réduit à moins d'un millimètre, de sorte que le poids total reste inférieur à une livre : exactement 491 grammes. $A$ part un petit éclat ancien de la tôle de bronze, en haut de la bombe, et à part, sur le côté gauche, le classique " coup de godet" de la drague, l'ensemble est en parfait état de conservation. La patine, mince et presque transparente, apparaît partout de couleur fauve, seulement un peu plus foncée du côté interne. Sans doute le marinier crut-il bien faire en tentant de nettoyer l'extérieur ; il est providentiel qu'il y ait rencontré tant de difficulté qu'il y renonça bientôt, de sorte que son action regrettable n'a causé que peu de dommage. Deux petits trous ronds, percés de l'intérieur, par percussion, se remarquent vers

(1) M. Brunand, secrétaire du Groupe d'études historiques de Verdun, et notre confrère M. G. Rabouhan sont aimablement intervenus pour que le Musée de Chalon puisse recueillir l'objet. les tempes, ils ont pu servir soit à l'adaptation d'une jugulaire, soit a celle de paragnathides, comme on en voit sur les têtes sculptées des guerriers salyens d'Entremont et sur de nombreux casques romains.

Ce casque possède une réplique au Musée des Antiquités nationales de Saint-Germain-enLaye (fig. 2). Connue sous le nom de casque de Breuvannes, en raison de la localité de la Haute-Marne où elle fut exhumée en 1882 dans un ancien lit du Flambart, petit affluent de la Meuse, elle a été fort bien étudiée alors par E. Flouest ${ }^{2}$ dont la description s'applique trait pour trait au nouveau casque chalonnais. L'auteur remarque qu'un "simple calotte métallique, fondue d'une seule pièce, a été étendue, amincie et finalement transformée par un marteleur habile en un couvre-chef de combat d'une forme très régulière. On s'étonne seulement qu'il a paru suffire de lui laisser l'épaisseur d'une feuille de papier au sommet de la bombe, alors que la protection du crâne semblait réclamer sur ce point de plus sûres garanties ". Mais, ajouterons-nous, les casques de ce type, comme le constata plus tard Décheletle, devaient être doublés par une forte calotte de cuir dont on atlendait un supplément de sécurité trìs appréciable. Il en allait d'ailleurs pareillement pour tous les casques romains et du haut Moyen Age, qu'ils fussent en fer ou en bronze, ainsi que l'ont indiqué Mme G. FayderFeytmans et M. A. France-Lanord, à propos du casque mérovingien de Trivières ${ }^{3}$. Rappe-

(2) E. Flovest, Anliquilés gauloises découvertes dans le departement de la Maute-Marne, in Mém. de la Soc. nat. des Anliquaires de France, t. 43, 1882, p. 48. C. F. Frh. von Lipperieide, Antike Helme, 1896, p. 2.5 ; et DEcheletre, Manuel, t. IV, p. 1164. La fouille a donné aussi un pendant d'oreille hallstattien en forme de croissant.

(3) G. Falder-Feytmans el A. France-Lanohd, Le casque mérovingien de Trivières, extr. de la Rev. belge d'Archéologie el d'Ilisloire de l'Art, t. XX, 1951, p. 271. 


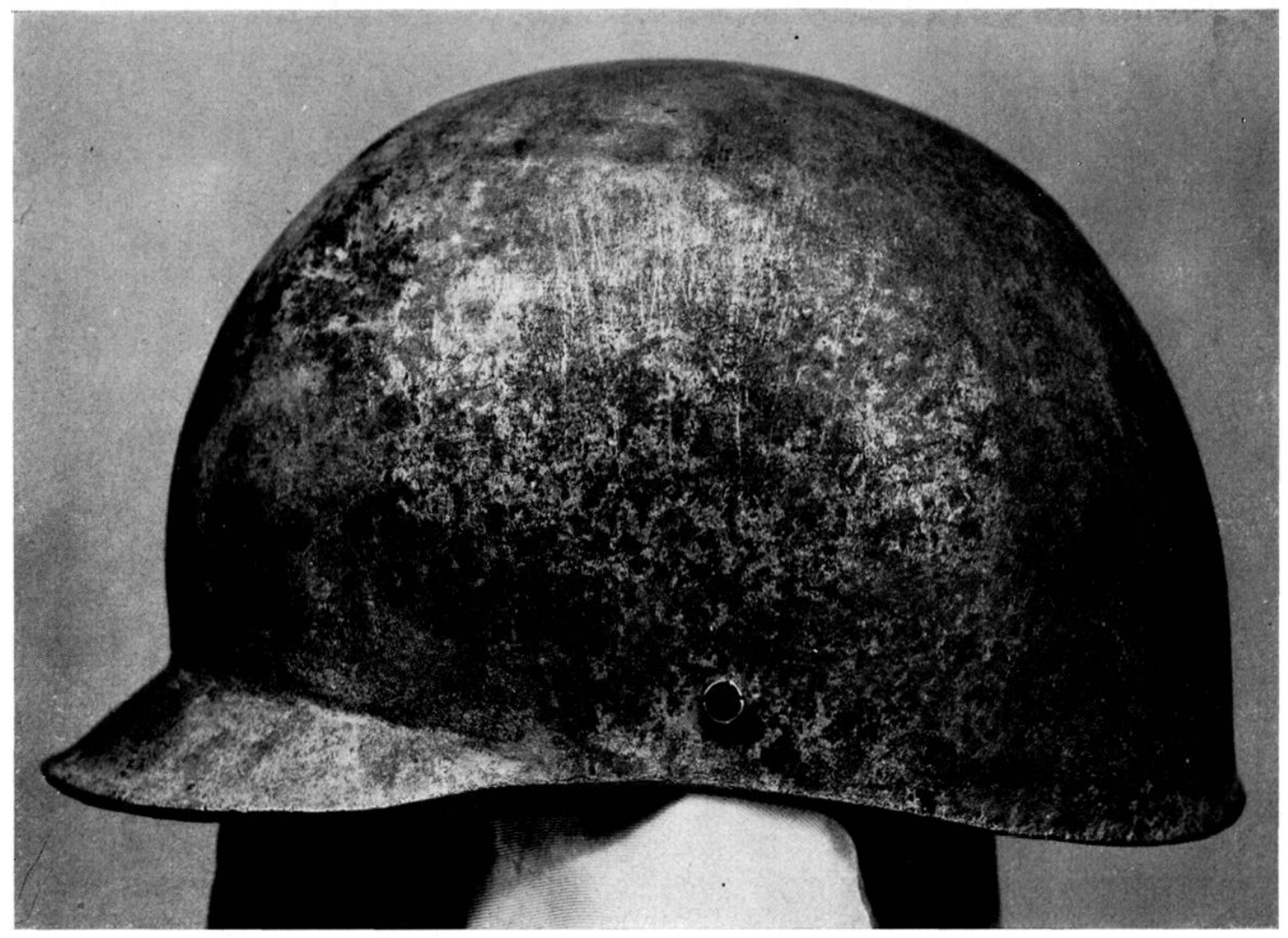

1 a. Casque dragué dans le Doubs prés de Ciel (Saône-el-Loire). Nusée de Chalon-sur-Saòne. Côté droit.

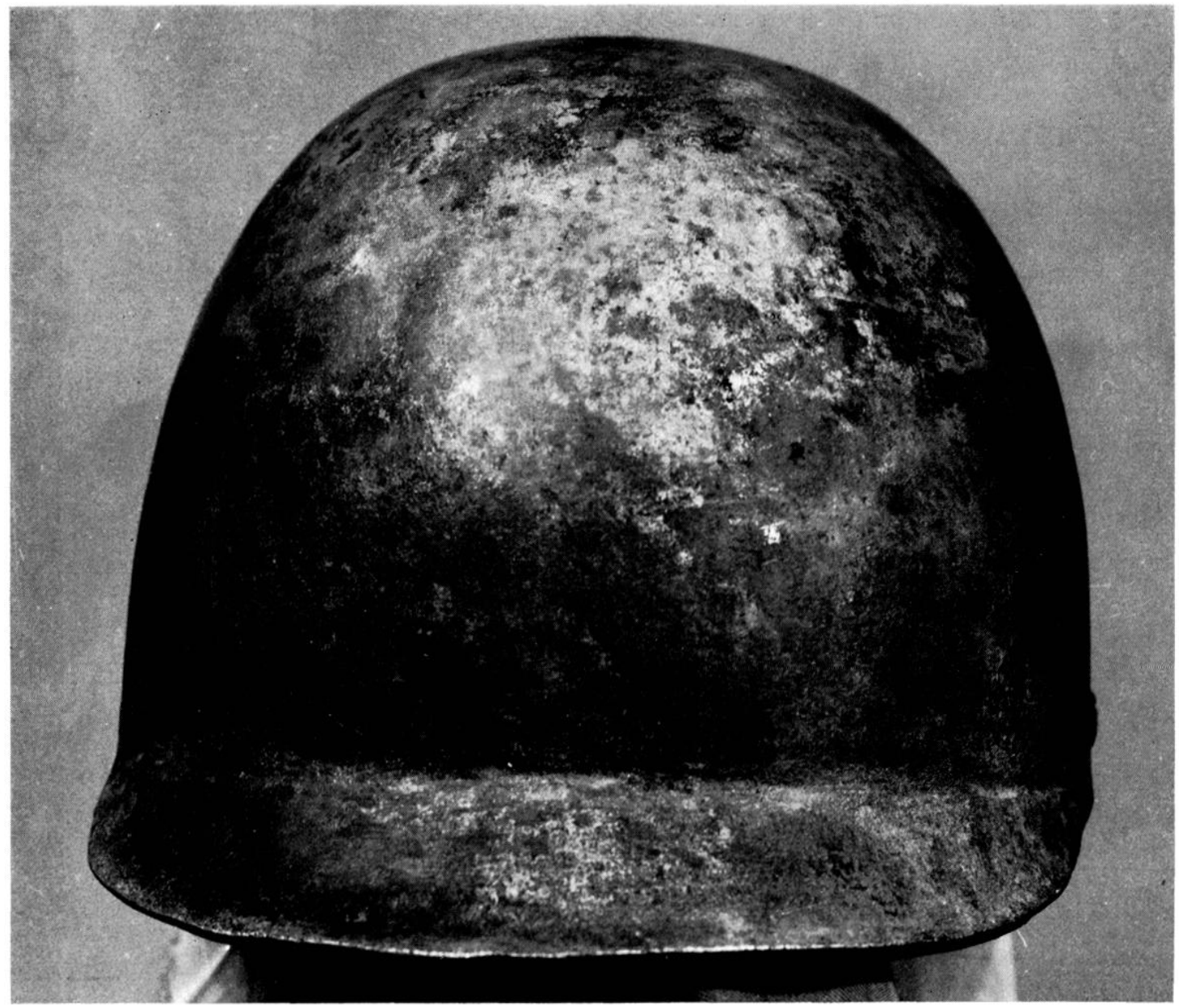

1 b. Casque dragué dans le Doubs près de Ciel (Saòne-et-Loire). Nuscee de Chalon-sur-Saòne. Vue prise par derrière. 
lons, en outre, qu'au temps de l'Indépendance l'on faisait en Gaule grand emploi de casques tout en cuir : les sculptures de Grézan et de Saint-Chaptes le prouvent; et une survivance de cel usage est altestée sous le règne de Tibère par les bonnets de peau de moulons figurés sur l'are d'()ranget.

Cine seconde réplique de notre casque existe au Brilish Iluseum (fig. 3) ; elle provient des dragages de la Marne, dans les limiles du département du même nom. Les seules variantes sont conslituées d'une part, par une triple ligne de petits points bordant par-devant la partic inférieure frontale, et, d'autre part, par la présence d'un troisième trou, percé intentionnellement vers le couvre-nuque, comme il l'est d'ailleurs sur l'exemplaire de Breuvannes, alors qu'il manque à Chalon. On peut se demander si cetle troisieme perforation ne servait pas a fixer un ornement quelconque, a allacher une doublure ou bien à prolonger le couvre-nuque, comme on l'a supposé à propos d'une pièce analogue $e^{5}$.

Conformément aux proportions d'un cràne normal, où le pariétal est plus grand que le frontal, le dessin fourni par l'empreinte du pourtour inférieur du casque de Chalon montre que la partie la plus resserrée est celle du front et qu'il y a un élargissement vers l'occiput (fig. 4), c'est-à-dire du còté du couvre-nuque. Or c'est le contraire que Flouest avait nolé à Breuvannes.

Cependant, à part ces menus détails, le nouvel exemplaire ne présente pas beaucoup d'originalité. Son mérite principal est d'être parvenu jusqu'à nous sans blessure notable. Les deux autres casques, dont nous venons de parler, sont au contraire très déformés par des chocs violents et répétés, donnés anciennement sur la bombe, de sorte que leur profil apparait avec moins de certitude.

Par contre, il existait déjà des exemplaires parfaitement conservés et identiques au nòtre en Bavière où le casque de Straubing (fig. 5), retiré du lit du Danube à une quarantaine de

(4) Amy, Deval, Hatt, etc., L'Are d'Orange, suppt à Gallia, 1962, pl. 43.

(5) Maurice Locis, Un casque du II a âge du Fer ì Monlpellier, dans Gallia, XI, 1953, p. 307. $\mathrm{km}$. en aval de Ratisbonne, a été étudié par Reinecke ${ }^{6}$, et en France, à Belfort, où l'on voit le casque de Lacollonge exhumé depuis peu " sous une épaisseur d'alluvions de 2 m " parmi "des pieux de chène plantés en palissade", vestiges possibles de la bataille livrée par César a Arioviste, selon M. L. Lerat?.

Ces cinq casques, c'est-à-dire ceux de Breuvannes, de la Marne, de Straubing, de Belfort et de Chalon sont des armes défensives remonlant à une assez haute anliquité. Joseph Déchelette datail ce type de la première période

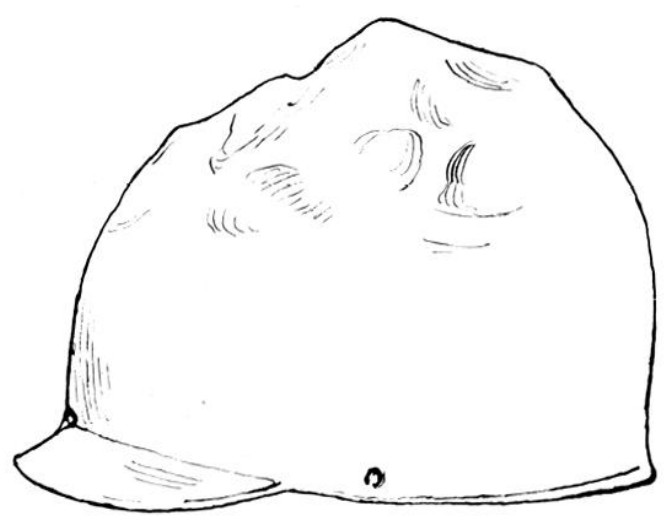

2. I.e casque de Breuvannes (IIaute-Marne), en bronze martelé, au Musce des Antiquités nationales de Saint(iermain-en-Laye, d'après E. Flouest. Long. 0.235; larg. 0,160 ; haut. 0,150 .

de La Tène ${ }^{8}$, et, suivant certains archéologues d'aujourd'hui, rien ne semblerait devoir faire réviser une telle chronologie; ainsi, pour M. Maurice Louis, ces " bombes " unies devaient être contemporaines des casques de Montpellier, des Martres-de-Veyre et de Majorque qu'il attribue aux $I^{e}-111^{e}$ s. avant J.-C.9. Nous

(6) Paul RE⿺xcks, Ein frühlaiserzeillicher Bronzehelm aus der Donau bei Slraubing, dans Germania, 29, 1951, p. 37 a 44. La dimension de ce casque sont : long. 0,23 ; larg. 0,187 ; haut. $0,142 \mathrm{~m}$.

(7) L. Lenat, Rapporl dans Gallia, 1960, fasc. 2, p. 261-262. Nous remercions M. Lelat pour les renseignements qu'il nous a procurés sur les casques de Belfort et de Straubing ainsi que .11 . le prof. Le Gall et II. R. Joffroy pour les indications qu'ils nous ont fournies sur les casques de Saint-Germain.

(8) Díchlatrte, Manuel, 1 re éd., p. 1165.

(9) Maturice I.otis, Gallia, XI, 1953, p. 307. Au Musée de Saint-Germain, le casque de Breuvannes est toujours classé à la Tène I. 


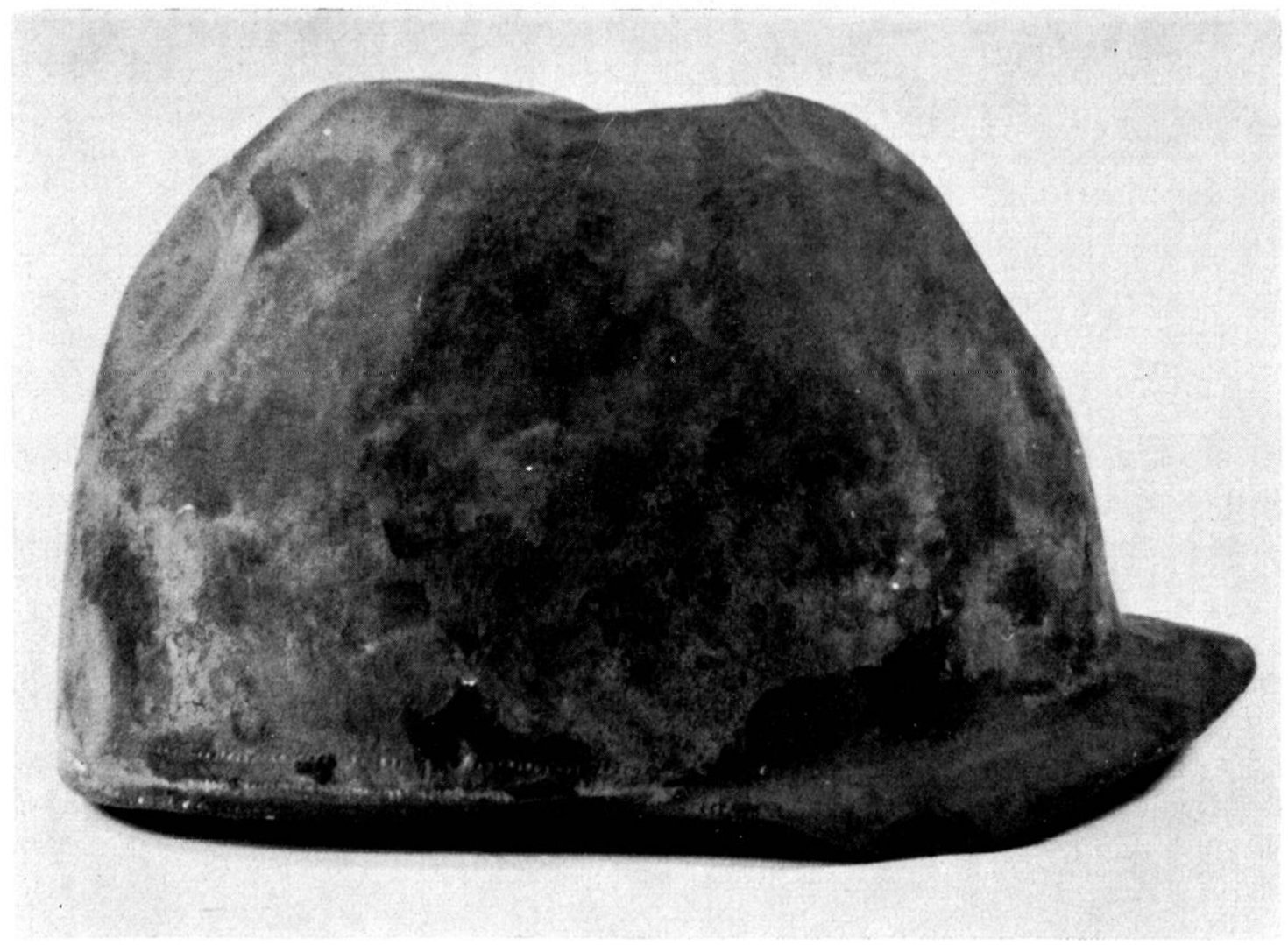

3 a. Casque de la Marne au British Museum, d'après le moulage du Musce des Antiquités nationales. Vue prise du còté gauche. (Phol" René Joffroy).

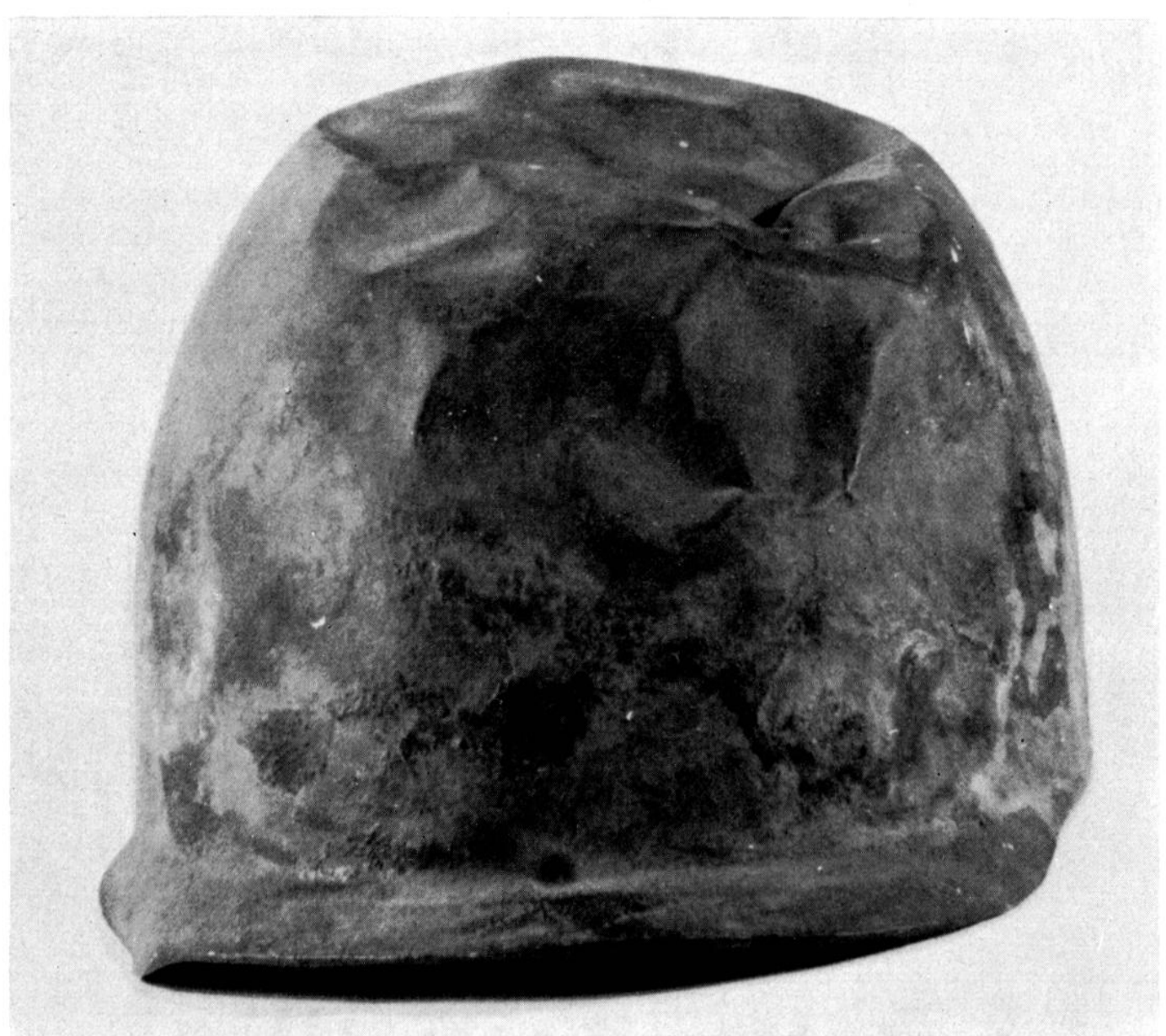

3 b. Casplue de la Marne au British Museum. Vue prise du coté du couve-nuque, d'apries un moulage. (Pholo René Joffroy). 


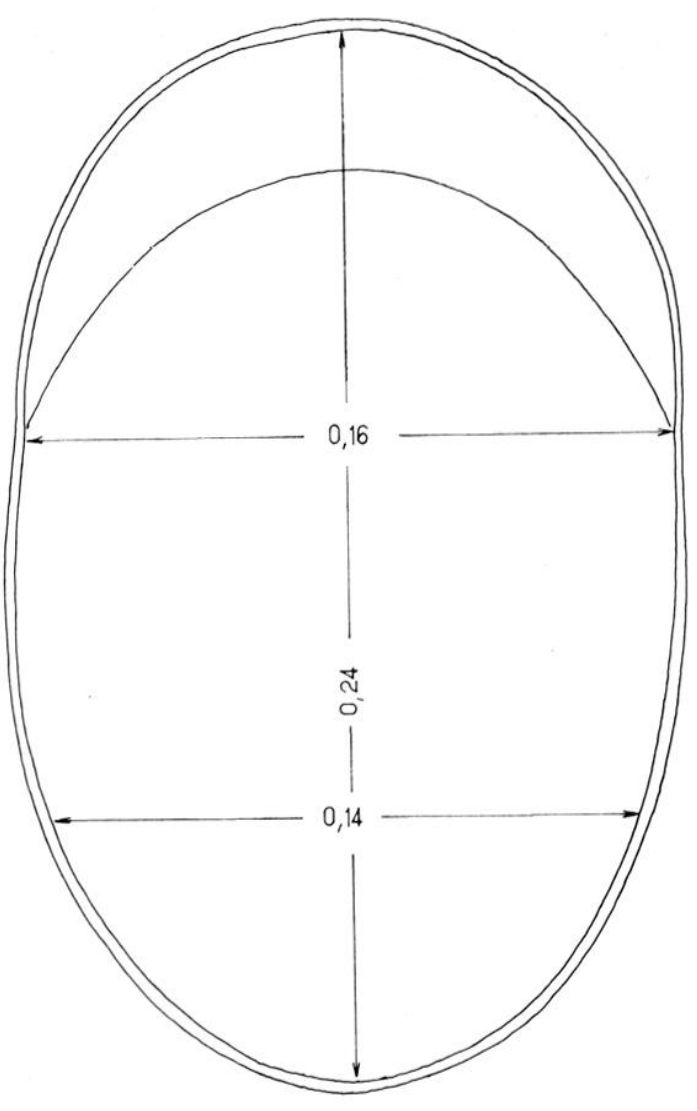

4. Casque du Musée de Chalon. Dessin de la base.

devons toutefois ajouter que Reinecke, suivi par M. le doyen Lerat, considère les casques de Straubing et de Lacollonge comme des armes romaines susceptibles de remonter à l'époque d'Auguste seulement, sans qu'il soit possible, il est vrai, de songer à une date plus récente ${ }^{\mathbf{1 0}}$.

Nous sommes en présence de casques dont les modèles ont été conçus dans les ateliers italo-grecs de la péninsule. Quelques spécimens étaient pourvus d'assez jolis ornements, comme celui de Mannheim qui comporte, au-dessus du couvre-nuque, à la base de la calotte, une étroite frise géométrique ciselée. ${ }^{11}$ Les prototypes importés en Gaule ont pu être imités fort habilement par l'industrie locale, car les Celtes étaient de très adroits métallurgistes. Il suffit d'examiner les situles et les chaudrons nombreux de la Tène III ${ }^{12}$ exposés au Musée de

(10) L. Lerat, loc. cit.

(11) Díchelette, fig. 489, 1.

(12) L. Armand-Calliat, Chaudrons el vases en
Chalon pour se convaincre de la dextérité avec laquelle des artisans, établis sans doute de ce còté des Alpes, savaient façonner au marteau des tôles de bronze extrêmement minces et cependant résistantes.

Les casques gaulois sont toujours rares; cela tient au fait que les Celtes, comme plus tard les Mérovingiens, avaient l'habitude de combattre la tête nue ou bien couverte d'une simple coiffure en peau, ainsi que nous venons de le dire. Quant aux casques en bronze pro-

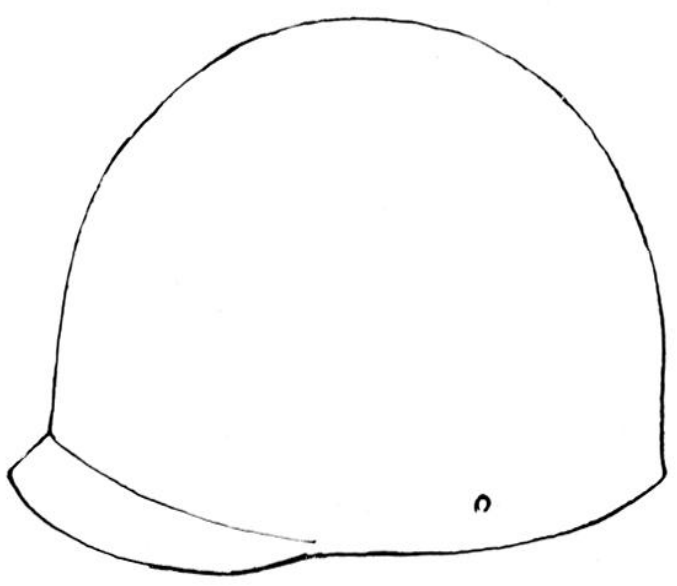

5. Casque de Straubing, d'après P. Reinecke. Long. 0,230 ; larg. 0,187 ; haut. 0,142 .

prement, romains, on en a peu, à ce qu'il semble, recueilli en Gaule qui soient sûrement datables de la République ou du règne d'Auguste; ils sont généralement de moins haute époque ${ }^{13}$.

Chez les Celtes, seuls les chefs arboraient le casque métallique, bien moins d'ailleurs pour se protéger le crâne contre les coups de leurs adversaires que pour se distinguer des guerriers placés sous leurs ordres. N'ayant normalement, en fait d'armes défensives, que le bouclier,

bronze de La Tène III, dans le Bull. archéologique du Comite des Travaux histrriques, janvier 1946.

(13) El ce sont souvent des casques non de combat, mais d'apparat, comme celui de la coll. Millon trouvé à Chalon (Cat. de la coll. Millon par Décheletle, 1913, p. 253 et pl. XLIII et XLIV) ou des objets votifs, voire de théatre, comme celui qui a été trouvé vers 1900 a Autun sur l'emplacement présumé d'un sanctuaire de Mars (Bullot, Mem. de la Soc. eduenne, t. 29, 1901, p. 357 ; A. France-Lanord, Revue des Arts, 1959, p. 112). 
les Gaulois tenaient à leur glorieuse "nudité " afin de mieux affirmer leur bravoure devant. l'enneminit.

$\Lambda$ ce propos, il n'est pas sans intérêt de remarquer que l'endroit précis où le casque chalonnais a été dragué avait déjà retenu notre attention par des découvertes archéologiques. En 1960, nous avions eu l'occasion de signaler que "le Doubs possédait un passage au port de La Colonne, lieu-dit de Ciel, où M. le Dr Maurice Carlot a recueilli des armes celtiques $»^{15}$. Depuis lors, le Musée IDenon y a glané à son tour, sur la drague, des pointes de lance de La Tène, on y a aussi trouvé des armes de bronze. Il est fort vraisemblable que des engagements ont eu lieu sur ce passage aux temps lointains de la protohistoire et a l'aube de l'histoire. C'était une coutume che\% les Celtes de livrer bataille sur les gués, de façon à ce que le dieu du fleuve décide entre les

(14) Juldux, Hist. de la Gaule, t. II, p. 196, citant Diodore, $\mathrm{V}, 30,3$, remarque que par "nudite ", il faut entendre absence de cuirasse et non de vêtements, cf. Raymond Laxtis, Revue archéologique, 1963, 2, p. 94 .

(15) L. Armavd-Candiat, Le Chalonnais à l'époque de La Tène, dans le Bulletin archéologique du Comité des Trancurr historiques, 1960, p. 122. adversaires; et cetle habitude revécul encore après les grandes invasions, car elle appartenait, également aux Germains. Les observations que nous avons personnellement faites, tout au long du cours de la Saône, à Anse (près de Villefranche), à La Truchère (au sud de T'ournus), à Allériot et à Gergy (en amont, de Chalon) semblent corroborer sur ce point les lémoignages littéraires et les hypothèses des archéologues ${ }^{16}$. Sans donc exclure la possibilité d'un naufrage de bateau ou bien celle d'un vulgaire accident survenu à quelque cavalier tentant de franchir la rivière en temps de crue, nous pensons que le casque récemment entré au Musée de Chalon a pu être perdu au cours d'un combat vers les limites des Eduens el des Séquanes, ces deux peuples rivaux qui se disputient longtemps le passage des fleures. Il se pourrait aussi que ce point ait été défendu contre un délachement de troupes romaines lors de la conquête des Gaules, si l'on admetlait pour le casque de Ciel la datation la plus basse et si on lui reconnaissait une origine italienne.

\section{Armand-Galdiat.}

(16) Cf. ce que nous écrivons dans Gallia, XV, 1957 , p. 141-14\%. 